\title{
Reflets
}

Revue d'intervention sociale et communautaire

\section{Un filet de sécurité sociale troué : la métaphore de la courtepointe pour raconter des histoires de femmes débrouillardes vivant en situation de pauvreté et qui doivent composer avec une santé fragile}

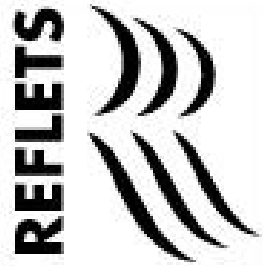

\section{Lise Savoie, Hélène Albert et Isabel Lanteigne}

Volume 22, numéro 2, automne 2016

La recherche et l'intervention en travail social en contexte francophone minoritaire au Canada

URI : https://id.erudit.org/iderudit/1038970ar

DOI : https://doi.org/10.7202/1038970ar

Aller au sommaire du numéro

Éditeur(s)

Reflets, Revue d'intervention sociale et communautaire

ISSN

1203-4576 (imprimé)

1712-8498 (numérique)

Découvrir la revue

Citer cet article

Savoie, L., Albert, H. \& Lanteigne, I. (2016). Un filet de sécurité sociale troué : la métaphore de la courtepointe pour raconter des histoires de femmes débrouillardes vivant en situation de pauvreté et qui doivent composer avec une santé fragile. Reflets, 22(2), 44-68. https://doi.org/10.7202/1038970ar
Résumé de l'article

Cet article présente des résultats d'une recherche qualitative sur la santé des femmes vivant en situation de pauvreté en régions rurales francophones du Nouveau-Brunswick. C'est à partir de la métaphore de la courtepointe que les auteures ont étudié les contextes dans lesquels les femmes rencontrées développent des stratégies de prise en charge de leur santé. Les résultats soulèvent des enjeux d'ordres collectif et politique, puisque la pauvreté chez les femmes ne constitue pas un problème individuel. Il s'agit d'un phénomène social qui commande des actions structurelles dans un contexte où le filet de sécurité sociale est troué.
Tous droits réservés (C) Reflets, Revue d'intervention sociale et communautaire, 2017 cest próéé par la loi sur le droit d'auteur. L’utilisation des services d’Érudit (y compris la reproduction) est assujettie à sa politique d'utilisation que vous pouvez consulter en ligne. 


\title{
Un filet de sécurité sociale troué : la métaphore de la courtepointe pour raconter des histoires de femmes débrouillardes vivant en situation de pauvreté et qui doivent composer avec une santé fragile
}

\author{
Lise Savoie \\ Professeure, École de travail social, Université de Moncton \\ Hélène Albert \\ Professeure, École de travail social, Université de Moncton \\ Isabel Lanteigne \\ Professeure, École de travail social, Université de Moncton
}

\section{Résumé}

Cet article présente des résultats d'une recherche qualitative sur la santé des femmes vivant en situation de pauvreté en régions rurales francophones du Nouveau-Brunswick. C'est à partir de la métaphore de la courtepointe que les auteures ont étudié les contextes dans lesquels les femmes rencontrées développent des stratégies de prise en charge de leur santé. Les résultats soulèvent des enjeux d'ordres collectif et politique, puisque la pauvreté chez les femmes ne constitue pas un problème individuel. Il s'agit d'un phénomène social qui commande des actions structurelles dans un contexte où le filet de sécurité sociale est troué.

Mots clés : femmes, santé, pauvreté, ruralité, débrouillardise sociale, filet de sécurité sociale, métaphore de la courtepointe

\section{Abstract}

This article presents the results of a qualitative research on the health of women living in poverty in rural francophone regions of New Brunswick. The quilt metaphor was used to illustrate the coping strategies that they develop when facing health issues. The findings raise collective and political concerns, as women's poverty is not an individual 
problem, but a social phenomenon that requires structural changes in a context where the social safety net is inadequate.

Key words: women, health, poverty, rurality, resourcefulness, social safety net, quilt metaphor

Dans son discours souvent renouvelé, l'État canadien a affirmé son intention de réduire, voire d'enrayer la pauvreté. Pourtant, Campagne 2000 indique une augmentation sensible de la pauvreté infantile au cours des vingt-cinq dernières années, et ce, malgré un objectif de l'éradiquer à l'aube de l'an 2000. Cette pauvreté des enfants reflète une situation sociale plus large d'iniquité dans la redistribution de la richesse. Or, l'écart grandissant entre les riches et les pauvres (Fahmy et Venne, 2014, p. 134) exige que l'État prévoie des mesures de redistribution plus justes visant à l'atténuer. En ce sens, selon Wilkinson et Pickett (2013), plus cet écart est grand, voire plus la société est inégalitaire, plus les problèmes sociaux risquent d'être importants. Cantillon (2014) indique que " toute politique nécessite une amélioration de la capacité redistributive et les dépenses sociales prévues doivent favoriser les pauvres" (p. 76). Or, bien que le Canada ait des mesures de redistribution, celles-ci ne réussissent pas à diminuer la pauvreté (Boismenu, 2008). C'est donc dire que le filet de sécurité sociale est troué, affectant ainsi la capacité des personnes en situation de pauvreté d'améliorer leurs conditions de vie. Par surcroît, plus la situation de pauvreté perdure, plus cela engendre des problèmes de santé, d'exclusion et d'isolement social, rendant davantage difficiles les possibilités de sortir de la pauvreté (Raïq et Plante, 2013).

Au Canada, les femmes sont plus nombreuses que les hommes à expérimenter la pauvreté et elles sont plus à risque de devenir pauvres (Sharma, 2012). Le NouveauBrunswick n'échappe pas à cette tendance, la population la plus pauvre étant composée majoritairement de femmes, dont $47 \%$ gagnent moins de 20000 \$ par année (Gouvernement du Nouveau-Brunswick, 2012). Les principales causes de la pauvreté des femmes sont liées à l'inégalité du marché du travail ${ }^{1}$ (Yerochewski et Fortier, 2013; Reid et Tom, 2006), au divorce ou à la séparation (Raïq et Plante, 2013) ou encore à la gestion séparée des comptes des couples qui sont de petits salariés, la femme écopant souvent de la plus large part des dépenses liées au ménage (Yerochewski et Fortier, 2013).

Le présent article découle d'une recherche qualitative menée en 2014-2015 sur la question de la santé des femmes vivant en situation de pauvreté en régions rurales francophones du Nouveau-Brunswick. Cette étude a permis, entre autres, d'apprécier 
"Pour explorer les sphères de vie dans lesquelles les femmes développent des stratégies de prise en charge de leur santé, les auteures, reprenant la métaphore de la courtepointe ..., analysent leurs façons de retisser les mailles d'un filet de sécurité sociale troué."

les stratégies que mettent en place les femmes pour prendre en charge leur santé. La littérature révèle qu'il existe un lien étroit entre un faible statut socioéconomique et un mauvais état de santé (Raphael, 2010). La spécificité de cette recherche réside dans les contextes sociaux particuliers dans lesquels se retrouvent les participantes : elles ont un niveau d'alphabétisme peu élevé, vivent en situation linguistique minoritaire, et ce, en régions rurales. Pour explorer les sphères de vie dans lesquelles les femmes développent des stratégies de prise en charge de leur santé, les auteures, reprenant la métaphore de la courtepointe (Aptheker, 1989 et Green, 2013), analysent leurs façons de retisser les mailles d'un filet de sécurité sociale troué.

Pour permettre de bien comprendre les éléments du contexte néobrunswickois dans lequel cette recherche s'inscrit, les auteures présentent brièvement ici l'évolution récente quant au développement des politiques sociales au Nouveau-Brunswick.

\section{Le filet de sécurité sociale néobrunswickois}

L'arrivée sur la scène politique en 1960 du premier ministre libéral Louis J. Robichaud, suivie de la mise en ouvre de son programme Chances égales pour tous, a transformé l'univers des politiques sociales au Nouveau-Brunswick et la conception de l'aide accordée aux populations plus vulnérables (Provencher, 2004). Les politiques sociales, qui ont été élaborées par son gouvernement pour une province où la ruralité et la francophonie étaient trop souvent synonymes de pauvreté, cherchent à réduire les écarts évidents entre les régions rurales du Nouveau-Brunswick, dont plusieurs sont majoritairement francophones, et les régions urbaines de la province à majorité anglophones. Le programme Chances égales pour tous, investi d'une "préoccupation de justice sociale », visait à ce que les citoyennes et les citoyens de cette province aient un droit d'accès égal aux programmes et aux services en matière de santé, d'éducation et de services sociaux sans égard à la région habitée ou à la langue parlée (Young, 2001, p. 28), et ce, dans une visée d'universalisation des programmes sociaux, s'inscrivant ainsi dans la mouvance de l'État-providence.

Cependant, dès les années 1970 , le modèle providentialiste commence à être remis en question (Rosanvallon, 1992). De nombreux groupes s'élèvent pour contester ce qu'ils nomment " les excès " de l'État-providence. En 1974, le gouvernement 
conservateur de Richard Hatfield « réforme l'aide sociale pour rendre les assistés sociaux plus «indépendants». Des projets d'intégration au marché du travail sont créés pour les personnes "aptes au travail ${ }^{2}$ " (Morel, 2002, p. 89). La province redéfinit sa forme d'assistance en écartant le critère de besoin pour favoriser celui de l'employabilité. L'aide accordée par l'État devient moins généreuse et par ailleurs de plus en plus conditionnelle.

Au début des années 1980, une augmentation significative de la pauvreté occasionnée par une récession économique importante est observée dans tous les pays industrialisés. Cette situation entraîne des mesures plus restrictives en matière d'aide sociale, d'inspiration néolibérale et à tendance résiduelle (Provencher, 2004). C'est en 1995 que le gouvernement fédéral modifie sa contribution aux provinces et diminue les paiements de transfert à ces dernières, affectant ainsi les moyens dont les provinces disposent pour assurer la prestation de programmes et de services (Boismenu, 2008) en matière d'éducation, de santé et de services sociaux. Non seulement les provinces recevaient-elles moins en transferts du fédéral $^{3}$, mais également elles n'étaient par ailleurs plus soumises de la même manière aux règles et aux normes nationales en matière de santé et de services sociaux, ce qui a entraîné, par exemple, de grandes disparités en ce qui a trait aux taux d'aide sociale accordés d'une province à l'autre (Moscovitch et Thomas, 2015).

Ainsi, l'effritement de l'État-providence et la montée de l'État néolibéral ont contribué à l'affaiblissement du filet de sécurité sociale, participant ainsi à la précarisation des conditions de vie des groupes les plus vulnérables. Les femmes en situation de pauvreté en ont été particulièrement affectées. Recevoir de l'aide ou des prestations est devenu plus difficile (Green, 2013), les critères d'admissibilité étant plus restrictifs.

\section{La pauvreté des femmes}

"La conception qui voudrait que la pauvreté soit liée à des facteurs individuels semble creuse puisqu'elle écarte les éléments de contextes et les rapports sociaux de domination qui $y$ contribuent..."
La pauvreté comporte plusieurs visages. Or, celui auquel on fait davantage référence correspond aux personnes qui reçoivent de l'aide sociale. Contrairement à la croyance populaire, cependant, la plupart des personnes en situation de pauvreté travaillent (Yerochwski et Fortier, 2013). La conception qui voudrait que la pauvreté soit liée à des facteurs individuels semble creuse puisqu'elle écarte les éléments de contextes et les rapports sociaux de domination qui y contribuent (McAll, 2008). Ainsi, les causes de la pauvreté ne résident pas dans la sphère individuelle, mais 
elles trouvent leurs racines dans des sphères sociales, politiques et économiques. La conjugaison de facteurs ou de conditions (le fait d'être une femme, la monoparentalité, la ruralité, l'accès ou non à des services de garde, etc.) accentue la pauvreté et sa durée. Par ailleurs, plus une personne vit longtemps dans la pauvreté, plus il devient difficile pour elle de s'en sortir (Raïq et Plante, 2013). Bien que souvent occulté, le visage féminin de la pauvreté est une réalité prépondérante. En ce sens, au Canada, le marché du travail est fortement genré (Reid et LeDrew, 2013), les femmes forment la majeure partie des travailleurs pauvres et elles occupent majoritairement des emplois à temps partiel (Yerochwski et Fortier, 2013) et atypiques. À cet effet, Savoie et Gaudet (2013) précisent que les femmes en régions rurales ont des occasions limitées de se sortir de la pauvreté étant donné la difficulté à se trouver un emploi bien rémunéré.

La pauvreté des femmes engendre l'exclusion sociale, qui s'exprime par l'absence de participation, d'intégration sociale et de pouvoir (Madanipour, Shucksmith et Talbot, 2015). Selon Madanipour, Shucksmith et Talbot (2015), plusieurs facteurs contribuent à cette exclusion sociale : l'espace social (quartier habité et stigmatisation associée à ce lieu), l'espace économique (épargne difficile, accès limité au crédit), l'espace institutionnel (bureaucratie, traitement inégal devant la loi, accès aux politiques sociales), l'espace territorial (accès aux services, disparités régionales rurales urbaines, accès au capital culturel) et l'espace symbolique (invisibilité sociale, traitement différencié, préjugés et discrimination). Les risques d'exclusion augmentent selon les effets combinés des catégories sociales auxquelles les personnes appartiennent (Madanipour, Shucksmith et Talbot, 2015). De plus, selon Klein et Champagne (2011), « les processus menant à la pauvreté et à l'exclusion sociale ne sont pas identiques, mais ils convergent » (p. 35). L'exclusion sociale renvoie aux personnes marginalisées et suppose une rupture, une mise à distance et une désaffiliation (Castel, 2009). La pauvreté qui dure dans le temps conduit à l'exclusion sociale (Klein et Champagne, 2011). L'exclusion sociale qui dure dans le temps fait perdurer la pauvreté, rendant la sortie de ce cercle difficile (Raïq et Plante, 2013). Dans cette perspective, la pauvreté persistante entraîne des difficultés, dont l'isolement, le problème de l'accès à l'emploi, la dépréciation des qualifications à l'emploi et la maladie physique et mentale (Raïq et Plante, 2013; McAll, et collab., 2012). Les femmes en subissent les conséquences, puisqu'elles expérimentent la pauvreté de manière plus significative et plus intense et elles demeurent plus longtemps dans la pauvreté (Reid et LeDrew, 2013).

Wilkinson et Pickett (2013) soutiennent que les inégalités sociales ont un impact sur la santé. Par surcroît, Broussard, Joseph et Thompson (2012), dans leur étude sur 
"...en ce qui a trait aux mères pauvres, devant l'insécurité alimentaire générée par leur condition, elles font des choix altruistes, en faisant passer les besoins des autres devant leurs propres besoins, privilégiant, par exemple, l'alimentation et la santé des enfants, au détriment de leur propre santé..." les mères monoparentales pauvres, indiquent que la pauvreté chronique génère du stress, dont l'incidence est importante sur les problèmes de santé physique et mentale. Ces derniers précisent que des facteurs de stress ayant le plus d'effets négatifs sur la santé sont associés aux défis qui se posent face à l'aide sociale, l'emploi, l'éducation, les services de garde, le voisinage, le soutien social et l'étiquetage. Par exemple, en ce qui a trait aux mères pauvres, devant l'insécurité alimentaire générée par leur condition, elles font des choix altruistes, en faisant passer les besoins des autres devant leurs propres besoins, privilégiant, par exemple, l'alimentation et la santé des enfants, au détriment de leur propre santé (Savoie, et collab., 2016; Broussard, Joseph et Thompson, 2012).

\section{Les contextes sociaux : alphabétisme, minorité linguistique et ruralité}

La pauvreté des femmes s'inscrit dans des contextes sociaux qui s'entrecroisent et qui en colorent l'expérience. Aux fins de la présente recherche, les dimensions de contextes retenues consistent en la prise en compte du niveau d'alphabétisme, de la situation linguistique minoritaire et de la ruralité. En ce qui a trait à l'alphabétisme, Nutbeam (2008) explique qu'il existe un lien entre un faible niveau d'alphabétisme, un faible statut socioéconomique et l'état de santé des populations. Au-delà des habiletés de lecture et d'écriture mesurées, l'alphabétisme lié à la santé, ou health litteracy, « désigne les capacités à mobiliser ses compétences et ses connaissances pour obtenir, traiter, comprendre, évaluer et agir pour prendre des décisions de santé et ainsi maintenir une bonne santé " (Moreau, Savriama et Major, 2013, p. 94), ce qui permettrait, selon Nutbeam (2008), aux individus d'exercer un plus grand contrôle sur la prise en charge de leur santé. Concrètement, étant donné le lien étroit entre le niveau d'alphabétisme et le statut socioéconomique, la trajectoire de santé des individus en est influencée, les conséquences sur la santé pouvant se manifester de diverses manières, dont la durée d'hospitalisation, le taux de maladies chroniques ou encore le taux de mortalité (Moreau, Savriama et Major, 2013).

Au Nouveau-Brunswick, les francophones sont en minorité, puisqu'ils constituent près du tiers de la population. En ce qui a trait à la population francophone et acadienne 
au Nouveau-Brunswick, $67 \%$ possèdent un niveau d'alphabétisme inférieur à la moyenne canadienne, soit sous le niveau 3, qui constitue le seuil minimum pour fonctionner ou encore pour maintenir un emploi (Savoie, 2011). Dans ce contexte linguistique minoritaire, le niveau d'alphabétisme, conjugué au statut socioéconomique, influence l'accès aux services de santé. D'abord, le niveau de langue qui sépare le discours des professionnels de la santé de celui des personnes en situation de pauvreté et peu alphabétisées constitue une barrière importante dans la prise en charge de sa santé (Drolet, et collab., 2014). De plus, au-delà du niveau de langue, c'est parfois la langue maternelle elle-même qui est évacuée au profit de l'accès plus rapide à un service ou tout simplement par déférence. Cependant, exprimer sa situation de santé dans la langue de l'autre constitue un obstacle majeur à la communication et à l'affirmation de ses besoins, ce qui peut participer à l'augmentation du stress, à l'établissement d'un diagnostic pouvant être erroné ou encore à l'administration d'un traitement inapproprié (Drolet, et collab., 2014). À cet effet, des auteures et auteurs affirment que l'accès aux services de santé dans sa langue en contexte francophone minoritaire constitue un défi de taille (Drolet, et collab., 2015; Forgues et Landry, 2014).

À la lumière de ce qui vient d'être énoncé, il est possible d'apprécier en quoi l'état de santé en contexte francophone minoritaire est lié au statut socioéconomique et au niveau d'alphabétisme. Par ailleurs, la prise en compte de la ruralité ajoute aux défis qui se posent aux femmes en matière de santé. Au Nouveau-Brunswick, les communautés francophones et acadiennes se trouvent principalement en milieu rural, ce qui a une incidence sur leur accès aux services de santé, les ressources en matière de santé en milieu

"Le cumul des identités (femme, pauvre, peu alphabétisée, francophone, campagnarde) contribue au développement de préjugés affectant ainsi les rapports avec les services de santé surtout quand elles doivent se déplacer en milieu urbain pour $y$ accéder..." rural étant souvent limitées et parfois inaccessibles (Nayar, et collab., 2012; Peterson et Litaker, 2010). Bien qu'il existe des avantages au fait de vivre en milieu rural (soutien social, environnement paisible, lien d'appartenance), le sentiment d'isolement, le manque d'affiliation avec les institutions ou des personnes détenant du pouvoir et la dépendance aux autres pour les déplacements sont au nombre des défis que rencontrent les personnes aux prises avec des problèmes de santé qui vivent en milieu rural, et ce, de manière plus sensible quand ces dernières sont en situation de pauvreté (Milbourne, 2014). Le cumul des identités (femme, pauvre, peu alphabétisée, francophone, campagnarde) contribue au développement de préjugés affectant ainsi les rapports avec les services de santé surtout quand elles doivent se déplacer 
en milieu urbain pour y accéder (Madanipour, Shucksmith et Talbot, 2015). Rendre compte des interactions entre des éléments de contextes et l'expérience des femmes rencontrées suppose de recourir à une perspective théorique pour en construire le sens, ce que la métaphore de la courtepointe rend possible.

\section{La métaphore de la courtepointe pour rendre visible le quotidien des femmes}

Plusieurs chercheures ont utilisé la métaphore de la courtepointe pour représenter les réalités du quotidien des femmes (Apetheker, 1989; Koelsch, 2012). La courtepointe est composée d'un ensemble de bouts de tissu cousus de façon créative pour en faire un tout offrant une esthétique unique. Apetheker (1989) explore le quotidien des femmes dont les réalités et les identités sont diverses. Sa réflexion s'inspire du travail de femmes (artistes, travailleuses d'usine, travailleuses domestiques, etc.) dont l'écrivaine américaine Alice Walker. L'œuvre littéraire de cette dernière a consisté à rassembler des bouts d'histoires, tombés dans l'oubli collectif, de femmes noires du sud états-unien. Ces femmes créaient des courtepointes pour raconter des moments de leur histoire familiale et collective, question de matérialiser leurs récits. Ces courtepointes illustraient leur vécu, en rattachant des bouts de tissus provenant de vêtements de la famille et de la communauté, pour représenter des valeurs et des symboles culturels, et parfois même pour cartographier les routes à suivre menant à leur libération (Singh, 2010). Forte de cette inspiration, Apetheker (1989) analyse l'expérience de femmes à partir de nombreuses dimensions de leur quotidien, soit le travail de maintien des relations interpersonnelles (p. ex. fêtes, anniversaires), le travail émotionnel du care (p. ex. lire les pensées et les émotions des personnes accompagnées), le travail assurant le bien-être des enfants et des autres membres de la famille et le travail domestique (p. ex. achats, nettoyage). Cette auteure, préoccupée par l'invisibilité du travail quotidien des femmes, utilise la courtepointe pour mettre en valeur les nombreuses contributions de ces dernières. Koelsch (2012), quant à elle, utilise la métaphore de la courtepointe pour mettre en lumière les résultats de recherches féministes afin de représenter les éléments de vécu commun des femmes. Cela permet de tisser les histoires singulières des femmes interviewées pour dépeindre dans une courtepointe leur histoire commune.

Green (2013), comme d'autres chercheures, utilise la métaphore de la courtepointe afin de saisir les réalités vécues par des femmes en situation de pauvreté. Trop souvent, elles sont perçues comme paresseuses ou non productives. Pourtant, comme l'analyse à 
partir de la courtepointe l'illustre, ces femmes doivent trouver de nombreuses stratégies pour survivre dans ces situations de pauvreté. Elles font en ce sens un travail créatif similaire à celui de créer une courtepointe pour retisser les mailles laissées par les trous dans le filet de sécurité sociale (Green, 2013). Green (2013) propose donc d'explorer les dimensions suivantes pour comprendre le travail quotidien de survie : recourir à de nombreuses ressources, gérer de nombreuses responsabilités, accomplir de multiples tâches et combiner le travail de subsistance et le travail du care.

Or, dans la présente recherche, la façon d'appréhender la métaphore de la courtepointe pour comprendre la réalité de femmes en situation de pauvreté et ayant des expériences de santé difficiles, a consisté à créer une bordure à cette courtepointe. Ainsi, les dimensions structurelles, dont la pauvreté, l'alphabétisme, la ruralité et les enjeux linguistiques, conjuguées aux dimensions du travail quotidien de survie (Green, 2013) nommées précédemment, forment la structure, voire le cadre d'analyse de cette courtepointe. Il est à noter que d'autres éléments structurels, entre autres le capitalisme et le sexisme, auraient pu faire l'objet d'analyses pour illustrer des dimensions sociales inhérentes au phénomène de pauvreté et ainsi être ajoutés à la bordure. Toutefois, les dimensions retenues sont celles qui émanent du discours des femmes rencontrées. Or, à l'instar de Walker, ce travail d'analyse dépasse sa dimension rationnelle puisqu'elle exige une sensibilité nécessaire à la transformation du regard posé sur la vie de ces femmes afin d'élucider les capacités créatrices, voire la débrouillardise (Demazière, 2013), qu’elles mettent en œuvre pour survivre.

"La conjugaison des éléments de contextes et des stratégies de survie permet à la fois de constater les trous dans le filet de sécurité sociale et de saisir les pratiques de survie déployées par les fermmes pour retisser les mailles du filet, bien que le fil dont elles disposent soit la plupart du temps fragile et cassant."
C'est donc à partir de la métaphore de la courtepointe que la trajectoire des femmes a été analysée, puisqu'elle a permis de croiser des éléments de contextes (pauvreté, alphabétisation, ruralité, enjeux linguistiques) à des éléments de débrouillardise, voire de survie (c'est-à-dire recourir à de nombreuses ressources, gérer de nombreuses responsabilités, accomplir de multiples tâches, combiner le travail de subsistance et le travail du care). La conjugaison des éléments de contextes et des stratégies de survie permet à la fois de constater les trous dans le filet de sécurité sociale et de saisir les pratiques de survie déployées par les femmes pour retisser les mailles du filet, bien que le fil dont elles disposent soit la plupart du temps fragile et cassant. 


\section{L'orientation méthodologique}

C'est à partir d'une méthodologie qualitative basée sur des récits de vie et inspirée d'une perspective féministe que cette recherche a été réalisée. Les récits de vie permettent de "déployer des ensembles significatifs dans et par lesquels les parties usuelles prennent tout leur sens " (Grell, 2015 , p. 8) en tenant compte du contexte sociohistorique. Effectuer une recherche à partir d'une perspective féministe implique un positionnement de la part des chercheures en faveur des femmes, en les situant « au centre de [leur] investigation et en poursuivant un objectif de changement social» (Ollivier et Tremblay, 2000, p. 24). Cette perspective a influencé le choix du cadre théorique de la courtepointe puisqu'il s'agit de rendre compte du vécu singulier des femmes. Cette forme d'analyse renvoie à un regard qui cherche à comprendre le " point de vue des femmes, pour mettre en relief les formes de participation des femmes à l'histoire et à la politique " (Ollivier et Tremblay, 2000, p. 25).

Dans le cadre de cette recherche, deux régions acadiennes côtières et rurales du Nouveau-Brunswick ont été ciblées en raison de leur prédominance francophone, soit Gloucester, $85 \%$ et Kent, $73 \%$ (Réseau de développement économique et d'employabilité du Canada, 2006). Les chercheures sont allées à la rencontre de dix-sept femmes qui ont accepté de raconter des moments de leur expérience de vie témoignant de leur trajectoire sur le plan de la pauvreté et de la santé. Le parcours scolaire de la grande majorité d'entre elles a pris fin avant l'obtention de leur diplôme d'études secondaires. Les entrevues en profondeur ont eu lieu au printemps et à l'été 2014.

L'analyse des données a été réalisée selon trois approches, soit diachronique, compréhensive et thématique. L'analyse diachronique consiste à structurer les histoires des participantes en tenant compte des évènements vécus selon une perspective tem-

"Cette approche méthodologique a rendu saillant un vécu souvent invisibilisé de femmes francophones et acadiennes vivant en milieu rural, et ayant à composer avec une situation de pauvreté et un état de santé fragilisée." porelle, voire chronologique (Bertaux, 2010). L'analyse compréhensive vise à établir le lien entre les résultats de la recherche et les perceptions des participantes, et à imaginer, à l'intérieur des témoignages, les phénomènes qui sont racontés sous forme allusive, afin d'en faire l'interprétation et de comprendre les processus sociaux en jeu (Kaufmann, 2006). Enfin, l'analyse thématique permet de repérer, de regrouper et d'examiner les thèmes pertinents à la recherche qui ressortent des récits recensés (Paillé et Mucchielli, 2012). Cette approche 
méthodologique a rendu saillant un vécu souvent invisibilisé de femmes francophones et acadiennes vivant en milieu rural, et ayant à composer avec une situation de pauvreté et un état de santé fragilisée.

\section{Reconstruire les mailles du filet : le discours de femmes}

Les résultats de cette recherche ont mis en lumière les stratégies auxquelles des femmes ont recours pour rapiécer les mailles d'un filet de sécurité sociale qui est parsemé de multiples trous. C'est ici que les quatre dimensions identifiées par Green (2013) permettent de présenter le discours de femmes.

\section{Recourir à de nombreuses ressources}

Green (2013) souligne que les femmes doivent, de manière à compenser les manques dans le filet de sécurité sociale, consacrer du temps pour identifier, se procurer et maintenir des services, des biens et de l'argent. Dans cette perspective, préparer des repas économiques, trouver des activités de loisir gratuites pour les enfants, négocier le report des paiements, trouver un mécanicien qui peut réparer l'auto à faible coût, trouver des services de garde abordables, se déplacer de service en service et négocier des occasions pour ses déplacements, demandent le déploiement d'efforts importants souvent invisibilisés. Par exemple, Édith a eu recours à des sources de financement privé pour combler ses besoins financiers. Elle raconte :

" Pour avoir de l'argent pour mes médicaments, j’avais été me fourrer à la Finance (bureau de crédit privé). Parce que j’avais été à la banque, pis on m’a dit : « Non, vous ne pouvez pas avec votre salaire. Là, j’ai été à la Finance; je voulais avoir 2000 \$, pis je l'ai eu, parce qu'à la Finance, eux autres, ils carent pas (ils s'en foutent). Je l'ai eu et j'étais ben contente, mais c'était dur. C'est 27 ou $28 \%$ d'intérêts, tu sais. "

Or, d'une situation financière précaire, Édith se précarise davantage. Par ailleurs, à tous les efforts que ces activités exigent s'ajoute la nécessité de consacrer du temps pour développer un réseau de soutien et maintenir la réciprocité dans les échanges (Green, 2013). Un effet pervers de ce type de rapport réside dans le fait que certaines femmes vont jusqu'à refuser l'aide informelle offerte par leur réseau par souci de ne pas être capables d'en assurer la contrepartie (Offer, 2012). En ce qui a trait à Monique, qui est atteinte d'un 
cancer, elle dit accepter l'aide de ses proches, bien qu'elle n'ose souvent pas leur demander, se sentant une obligation de réciprocité. Émue, Monique raconte qu'elle se sent redevable envers sa famille : " Je me dis que ma famille m’a beaucoup aidée, pis on dirait comme j'ai... je leur dois là. Je leur dois, tu sais, je vieillis pis je leur dois encore. » Elle ajoute qu'elle ne peut pas refuser si sa famille lui demande quelque chose : "Je suis obligée de dire oui parce qu'ils ont fait ça pour moi, ben je suis obligée de dire oui. » Fatiguée à la fois par sa maladie et par sa condition de pauvreté (disposant d'un revenu de 537 \$ par mois), elle voit le poids de cette dette s'alourdir au fil du temps. Devant cette difficulté d'entretenir une réciprocité (Lisse, 2007), plusieurs femmes se retirent de relations (Offer, 2012), ce qui contribue, en l'absence de ressources sociales, à leur isolement (Stephens, Cameron et Townsend, 2014). Dans ces circonstances, elles se retrouvent

"Bien que leur réseau de soutien comprenne des membres de la famille et des amies et amis, il arrive que la possibilité de faire appel à ces personnes soit incertaine en raison de tensions qui peuvent se manifester dans les relations." donc dans des situations où elles ne peuvent compter que sur elles-mêmes (Stephens, Cameron et Townsend, 2014). Bien que leur réseau de soutien comprenne des membres de la famille et des amies et amis, il arrive que la possibilité de faire appel à ces personnes soit incertaine en raison de tensions qui peuvent se manifester dans les relations. Enfin, dans la perspective de maximiser les ressources du réseau, les femmes vont à l'occasion convertir une ressource dans une autre ressource (p. ex. une personne qui conduit la femme à ses rendez-vous médicaux peut aussi être celle qui va l'aider à trouver une gardienne à moindre coût) (Friedman et Graham, 2008).

\section{Gérer de nombreuses responsabilités}

Les femmes pauvres, en l'absence de moyens pour acheter des biens et des services, doivent consacrer beaucoup de temps pour coordonner l'organisation de leur vie familiale et domestique (Zilanawala, 2014; Green, 2013). Ce temps qu'elles doivent investir à ces fins empiète sur celui qui pourrait être consacré à leur mieux-être (p. ex. faire de l'activité physique pour prendre soin de leur santé). Zilanawala (2014) réfere à la notion de time poverty pour parler de cette réalité. En ce sens, plusieurs femmes rencontrées investissent beaucoup de temps pour négocier le report du paiement de factures et pour trouver les meilleures aubaines tant pour leurs médicaments que pour l'épicerie (Savoie, et collab., 2016). Bélinda et Ghislaine sont championnes en ce qui a trait au temps consacré à la recherche d'aubaines, ce qui restreint le temps qu'elles peuvent investir 
dans des activités qui favorisent leur bien-être. Celles qui travaillent à l'extérieur du foyer sont particulièrement affectées par cette exigence, puisque s'ajoute à leur temps au travail ce que Hochschild et Machung (2012) appellent le second shift (deuxième quart de travail). Quand il s'agit de femmes pauvres, Green (2013) réfere plutôt au unspoken shift (quart de travail ignoré). Wattis et James (2013), quant à elles, utilisent le concept de invisible responsabilities (responsabilités invisibles) en parlant de la responsabilité de maintenir l'équilibre, c'est-à-dire de planifier et d'organiser la sphère familiale et la sphère domestique. Devant le peu de temps qui leur reste objectivement, Green (2013) s'interroge à savoir comment elles arrivent à répondre à leurs propres besoins. Comme l'illustre la situation d'Olivia, une participante à la recherche menée par Green, elle a vécu de la violence conjugale et peine à jongler avec l'ensemble de ses responsabilités, à gérer les crises du quotidien et à assurer le maintien de ses rôles de mère et de pourvoyeuse. Il devient alors difficile pour elle de surmonter les effets de son vécu de violence. C'est donc faute de temps qu'elle en arrive à prioriser les autres, à son détriment. Une participante à la présente recherche, Bélinda, jeune mère monoparentale de deux enfants, dont un nouveau-né, doit jongler avec un deuxième travail pour joindre les deux bouts. C'est avec émotion qu' elle explique :

"Son lait "Similac" que j'achetais, ben, je l'ai mis sur ma carte de crédit. Il fallait que je trouve un deuxième revenu pour payer ma carte de crédit. J'ai travaillé de nuit à l'hôpital pour la payer. Ça, c'était difficile! Ce qui était le plus dur, c'est que tout le monde me disait que le père (son ex-conjoint) était au bar, qu'il payait la boisson à tout le monde, pis moi, j'n'avais même pas une caisse de lait. "

À la lumière des écrits des auteures et du discours des femmes, la gestion des nombreuses responsabilités qui leur incombent affecte la qualité et la quantité de temps dont elles disposent pour exister dans leur propre vie. Cependant, certaines ont développé des stratégies pour s'offrir du temps de loisir à moindre coût. Jeanne raconte :

«On va en ville, pis on arrête à des parcs. Moi, j’aime aller marcher pis prendre l'air, c'n'est pas de quoi qui coûte cher, mais c'est le fun. J'aime me promener au centre d'achat. Je regarde juste dans les magasins, mais ça ne fait rien. Tu espionnes d'une place à l'autre, tu regardes, tu sais, ça fait une sortie, boire ton café, rien d'extravagant. » 


\section{Accomplir de multiples tâches}

"Les fermmes pauvres sont souvent perçues comme non productives, mais leur réalité est tout autre. Elles sont occupées à accomplir de multiples tâches afin de répondre aux besoins de leurs proches."
Les femmes pauvres sont souvent perçues comme non productives, mais leur réalité est tout autre. Elles sont occupées à accomplir de multiples tâches afin de répondre aux besoins de leurs proches. À titre d'exemple, Friedman et Graham (2008) soulignent le fait de devoir jongler avec les paiements, téléphoner pour les factures en retard, transformer un logement peu salubre en un espace approprié pour la famille, aménager le logement à peu de coûts, nourrir des liens avec certaines personnes pouvant les aider, échanger des services, partager des connaissances avec d'autres, attendre en ligne. Pour ces auteures, ces multiples tâches illustrent la nécessaire productivité de ces femmes, alors que trop souvent elles sont considérées comme paresseuses et déviantes. Par ailleurs, Lisse (2007) ajoute : «Un temps important est consacré à la comparaison des prix et à la recherche de rabais. [...] Ces femmes pratiquent un système de consommation qui exige un travail de mémorisation et une recherche permanente du moindre coût. » (p. 735). Ainsi, à l'instar des exemples présentés plus haut qui évoquaient le temps consacré à la gestion de la pauvreté et de la santé, ces multiples tâches relèvent aussi de stratégies de survie. Or, non seulement elles sont productives, mais également elles possèdent l'art de la débrouillardise. En ce sens, la débrouillardise sociale se manifeste de différentes manières qui " améliorent l'existence, protègent du passage à une plus grande précarité, permettent de pourvoir aux besoins sans renoncer à quelques plaisirs de la vie. Cet « art de faire contribue à équilibrer les budgets et à aller, parfois, au-delà d'une économie de subsistance. Il permet de vivre et pas seulement de survivre. » (Lisse, 2007, p.736).

Bélinda témoigne de débrouillardise pour assurer, à son fils et à elle, un minimum de qualité de vie. Elle parle de marcher sur le bord de l'eau et de faire des pique-niques. En plus, pour agrémenter les sorties qu'elle fait avec son enfant, elle est prévoyante puisqu'elle achète des produits à rabais, tels que des boissons gazeuses, des bouteilles d'eau, des croustilles, qu'elle cache dans sa garde-robe. Elle raconte également comment se déroulent ces sorties :

«On va aller marcher. Là, l'été s'en vient, il va faire beau et on va aller marcher sur le bord de l'eau. Des fois, le mercredi, ils jouent de la musique. Ça fait que moi, j'aime écouter ça, pis c'est gratuit, tout est gratuit. Tout ce qui est gratuit, moi, j’y vais. Ça me coûte juste le gaz. Les feux d'artifice, on y va, 
peu importe où est-ce que c'est. Des fois, il y a des amies qui vont nous dire : "Ah! On va aller faire un pique-nique, venez! Bien, c'est ça que je fais. Ça ne coûte pas cher un pique-nique. Je veux dire, tu fais des sandwichs, des sousmarins, c'est toutes des choses que tu as chez vous là. Les restaurants, non là, mais c'est ça qu'on fait, des pique-niques, des choses faciles comme ça! »

Par cet extrait, il est possible de constater qu'outre le fait que les multiples tâches effectuées par ces femmes le sont dans le but d'assurer leur suivie, certaines sont déployées pour agrémenter leur existence. Donc, à toutes ces tâches qu'elles doivent accomplir pour survivre et pour vivre s'ajoutent celles qui touchent aussi le travail du care.

\section{Combiner le travail de subsistance et le travail du care}

Le travail du care revêt une dimension morale qui vise à contribuer à un monde meilleur et qui comprend de nombreuses activités " pour l'entretien de la vie humaine " (Paperman, 2015, p. 37). Cette forme de travail auprès des proches repose en majeure partie sur les femmes (Yeandle et Joynes, 2012) et est souvent non reconnue (Paperman, 2015) et, par surcroît, invisibilisée (le Blanc, 2009). En effet, comme le souligne le Blanc (2009), « les femmes sont particulièrement exposées à ce genre d'invisibilité » dans la mesure où elles sont généralement considérées " comme des instruments au service de la vie des autres » (p. 95). Par ailleurs, c'est à la fois par leur statut de mère, de conjointe, de sœur et de fille qu' elles sont appelées à adopter le rôle de soignantes (Austen, et collab., 2015). Carmel, qui déjà travaille comme aide-soignante, doit, en dehors de ses heures de travail, reproduire auprès de sa mère des tâches associées au care. Elle raconte : "Je travaille 44 heures par semaine. Ma mère est beaucoup malade. J'ai été obligée d'aller tous les soirs. Je sortais de chez le client à $8 \mathrm{~h}$, pis j'allais lui donner son bain. J'arrivais chez nous des fois à 10-11 h ». Il s'agit ici de ce que Hochschild et Machung (2012) nomment le second shift. Cette responsabilité à l'endroit de leurs proches se manifeste autant dans leur quotidien que dans des imprévus, notamment quand vient le temps de réagir à la maladie d'un proche ou à une situation d'urgence (Wattis et James, 2013). Malgré le fait qu'elles occupent ou non un travail rémunéré, elles demeurent responsables du care au sein de la famille. Pour faire écho à la notion de time poverty, quand ce travail du care relève de femmes en situation de pauvreté, l'empreinte de cet engagement auprès des proches dans leur vie et sur leur qualité de vie est particulièrement importante et laisse des traces en réduisant encore l'espace de temps sur lequel elles peuvent compter pour exister dans leur propre vie. Jeanne illustre bien cette réalité par le témoignage qui suit : 
"Mon frère, c'est comme mon enfant. Ça fait depuis que je suis petite que j'en prends soin. Ma mère est décédée, pis j'ai continué à en prendre soin. Je prenais soin de mon autre petit frère, qui est grand asteure. Bien, je m'occupe en premier de mon frère, pis après, de mon homme. Après, s'il reste de quoi, c'est moi, après..." "

Bien que le travail de subsistance combiné au travail du care puisse constituer un fardeau, certaines femmes, particulièrement les grands-mamans, y trouvent une source de bien-être. Maria s'occupe de son petit-fils, qui vient régulièrement dormir chez elle. Cet investissement de Maria auprès de son petit-fils contribue à la valoriser. Quant à Édith, elle raconte : "Ce qui m’a aidée beaucoup, c'est que je gardais mes petits-enfants. " C'était donc, pour elle, une source de réconfort, elle qui cumulait une situation de santé chronique et une précarité financière certaine.

\section{Ce que la courtepointe révèle}

Ce que la courtepointe révèle, c'est l'inefficacité du filet de sécurité sociale qui oblige les femmes à développer des stratégies de prise en charge. En ce sens, les dimensions structurelles que sont la pauvreté, l'alphabétisme, la ruralité et les enjeux linguistiques obligent les femmes à recourir à des stratégies individuelles pour tenir le coup dans leur contexte qui conjugue la pauvreté et des défis de santé. Elles utilisent des stratégies individuelles plutôt que collectives puisqu'elles sont confrontées, dans leur quotidien, à la survie. La débrouillardise individuelle de ces femmes a un effet pervers, du fait que non seulement elle n'agit pas sur les causes de leur situation, mais également elle contribue à

"Le croisement des stratégies de prise en charge avec les éléments de contextes sociaux permet également de reconnaître et d'apprécier ces femmes comme sujet de leur vie, à savoir qui elles sont, et non pas en vertu du statut d'objet qui leur est habituellement attribué. " invisibiliser davantage les inégalités. Cette courtepointe révèle aussi que ces femmes qui vivent en situation de précarité financière et de santé fragile sont débrouillardes, ingénieuses et persévérantes. Leur débrouillardise et leur ingéniosité renvoient à ce que Green (2013) nomme le quart de travail ignoré (unspoken shift), ou encore ce qu'il conviendrait aussi d'appeler le quart de travail invisible. Vus autrement, le premier quart de travail consiste en celui qui est lié au travail rémunéré, le deuxième quart de travail (Hochschild et Machung, 2012) réfere aux activités liées à l'organisation de leur vie familiale et domestique, et le troisième quart de travail est celui qui mobilise des actions 
liées à la survie en contexte de pauvreté. Le croisement des stratégies de prise en charge avec les éléments de contextes sociaux permet également de reconnaître et d'apprécier ces femmes comme sujet de leur vie, à savoir qui elles sont, et non pas en vertu du statut d'objet qui leur est habituellement attribué. Comme en témoignent les propos de Jeanne, qui n'a pas de diplôme d'études secondaires et qui travaille au salaire minimum :

" Je m’organise tout le temps, je n'ai pas le choix. J'ai appris ça jeune fille que si je voulais quelque chose... Par exemple, s'il faut que tu fasses nettoyer tes dents... Tes dents, c'est une partie importante de ta santé. C'est difficile, parce que c'est ça, si je sais que j'vais aller chez le dentiste, parce que j'ai eu ma date six mois d'avance, pis j'ai une idée de ce que ça va me coûter, bien j'essaie d'épargner. "

Au-delà des qualités de débrouillardise, d'ingéniosité et de persévérance que ces femmes manifestent, elles adoptent des pratiques en fonction de leurs besoins et de leurs moyens. En ce sens, en raison de leur condition de pauvreté et, par surcroît, de leur état de santé, la gestion financière du quotidien est particulièrement laborieuse (Savoie, et collab., 2016). Elles doivent économiser, chercher des aubaines, prioriser les paiements, emprunter, négocier et, de manière récurrente, se priver, ce qui contribue à ce que Zilanawala (2014) appelle le time poverty. Or, leurs stratégies les conduisent souvent devant des non-choix, alors qu'elles doivent choisir entre se nourrir (se procurer des aliments) et se soigner (se procurer des médicaments). Ces modes de débrouillardise qu'elles déploient sont nécessaires dans un contexte d'inégalités de genre, de classe sociale et de ruralité. L'intersection de ces dimensions structurelles pousse ces femmes à réagir dans l'immédiat, avec les moyens dont elles disposent, ce qui se traduit par le recours à des

"Pour leur permettre de tenir le coup, la spiritualité constitue aussi une stratégie significative,... qu'elles aient recours à la religion ou à la méditation, plusieurs femmes ont évoqué la pratique de ces formes de spiritualité dans leur vie." stratégies individuelles. La quantité de défis qu'elles ont à affronter en ce qui a trait à leur santé et à leur précarité financière dans leur quotidien fait en sorte qu'elles se retrouvent de manière récurrente en situation de crise, leur permettant difficilement de trouver les moyens de mobilisation collective pour agir de manière concertée sur les causes structurelles de leurs conditions de vie.

Pour leur permettre de tenir le coup, la spiritualité constitue aussi une stratégie significative, qui a été observée dans le discours des participantes. En effet, qu'elles aient recours à la religion ou à la méditation, plusieurs femmes 
ont évoqué la pratique de ces formes de spiritualité dans leur vie. Il s'agit soit de la méditation orientée dans une perspective de réconfort intérieur, soit de la prière formulée envers une entité extérieure à soi afin d'obtenir, pour l'autre ou pour soi, qu'un souhait, voire une demande, soit exaucé. Odette, qui se décrit comme une personne anxieuse, utilise le reiki comme forme de méditation. Carmel, pour sa part, trouve aussi du réconfort en faisant de la médiation : "Je vais dans ma chambre pis je fais ma méditation. J'aime beaucoup ça; ça me détend pis ça m’aide. " Dans les moments de désespoir de Léa, la prière semble avoir constitué une bouée pour elle :

" Je priais beaucoup. J'aurais été à la messe à toutes les fins de semaine. J'ai assez braillé [pleuré], j’ai assez braillé, je demandais au bon Dieu : « Pour l'amour de Dieu, il faut que tu m'aides parce que moi, je n'en peux plus. Maintenant, je ne vais plus à la messe, mais je prie les soirs, je suis une prieuse.»

Le discours de ces femmes dépeint le croisement des contextes dans lesquels elles se retrouvent et des stratégies auxquelles elles ont recours pour réaliser la courtepointe qui rassemble les différents morceaux de leurs réalités. L'illustration qui suit expose ce rapiècement qui rend visible tout ce qu'elles doivent investir en temps et en effort pour survivre, dans un contexte où elles doivent par surcroît composer avec des défis de santé.

Figure 1 — La courtepointe et lo quotidien des femmes

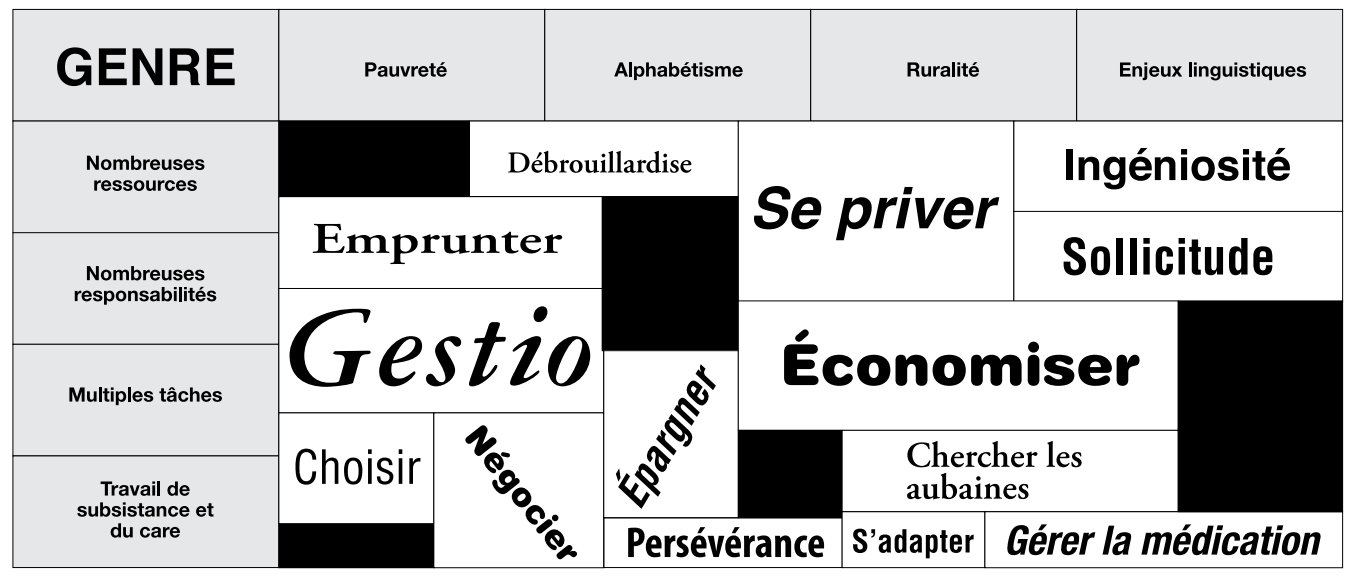

Ce labeur et ce courage qu'elles doivent déployer pour survivre sont tout à leur honneur. Il en demeure que leur situation précaire reste une réalité quotidienne avec 
laquelle elles doivent composer et qui comporte des coûts sur leur santé et sur leur qualité de vie. Quand tout ne tient qu'à l'effort individuel, sur quoi compter lorsque cet effort est insuffisant ou lorsqu'il n'est plus possible? La courtepointe comporte donc des trous.

\section{Outre le rapiècement, les trous qu'il reste}

Les réalités des femmes rencontrées sont complexes et tributaires des contextes sociaux dans lesquels elles se retrouvent. De manière générale, ce sont des femmes peu alphabétisées, vivant en milieu rural. Ces deux conditions limitent l'accès à de bons emplois bien rémunérés, avec des avantages sociaux les protégeant des risques naturels ou sociaux inhérents à la vie. Le risque de se retrouver en situation de pauvreté en est par ailleurs augmenté. C'est dans ces circonstances que deviennent davantage manifestes les trous du filet de sécurité sociale. L'histoire de Monique est parlante. En effet, bien que n'ayant pas de diplôme d'études secondaires, Monique réussissait à gagner modestement sa vie en travaillant comme serveuse dans un restaurant, à trente minutes de son domicile. Elle ne pouvait pas cependant contribuer à un régime d'assurance-santé privé. Or, quand elle a appris qu'elle était atteinte d'un cancer, outre la perte de sa santé, c'est son revenu et son niveau de vie qui en ont écopé, puisque, comme elle le dit si bien, elle est « tombée sur le bien-être ». Le montant reçu : 537 \$ par mois. Elle raconte :

"Ton mois, il est long en barnache quand il a trente et un jours. Tu les comptes tes jours! Tu as eu ta paie le $1^{\text {er }}$, pis le 2 ou le 3 , t’as payé ta facture d'électricité, ta facture de téléphone, t'as fait un petit peu d'épicerie, pis t'as plus d'argent... pis on est seulement le 3 ou le 4 , pis il y a trente et un jours..."

Le témoignage de Délia, qui est atteinte de diabète, d'arthrite et de haute tension artérielle, est touchant. Il reflète l'impact des trous dans le filet sur les conditions de vie des enfants :

"J'ai vu la petite aller à l'école avec rien dans sa boîte à lunch. Ça vient te chercher en s'il vous plaît. T'as une maison, t'as des enfants, il faut que tu vives. Mais j'ai tenu le coup! Ma fille disait : «Ben, Maman, c'est pas grave. Tu sais, des fois la maîtresse d'école donnait un $2 \$$ pour son lunch. Je n'ai jamais lâché pareil, pour l'amour des enfants. Mais j’ai eu honte, j’ai eu très honte...» 
"Ce qui est saillant dans ces propos de Délia, c'est que la pauvreté de la maman marque aussi la trajectoire de l'enfant, car, outre la boîte à dîner, c'est toute la stigmatisation qu'on peut imaginer qui accompagne le quotidien de l'enfant à l'école, et celui de la maman dans sa communauté."
Ce qui est saillant dans ces propos de Délia, c'est que la pauvreté de la maman marque aussi la trajectoire de l'enfant, car, outre la boîte à dîner, c'est toute la stigmatisation qu'on peut imaginer qui accompagne le quotidien de l'enfant à l'école, et celui de la maman dans sa communauté. Délia démontre une force certaine, mais on ne peut que supposer le stress additionnel qui émane de cette réalité et les effets sur sa santé déjà fragile. Or, on sent très bien que le stress est palpable dans la vie des participantes, ce qui a aussi été constaté dans les recherches de Savoie, et collab. (2016) et de Broussard, Joseph et Thompson (2012). Les tensions qu'Édith doit gérer entre payer des factures et nourrir sa famille sont manifestes. Elle raconte :

«Je ne te mens pas, la facture d'électricité là, pour manger, fallait que je passe par-dessus. Pis l'autre mois, ben j'payais la moitié, pis j’essayais ça de même. Mais là, je dois encore l'électricité, je dois encore... c'est un miracle qu'ils ne me l'ont pas coupée."

Ces témoignages de femmes dépeignent un système de protection sociale qui non seulement ne réussit pas à garantir le minimum pour vivre décemment, mais également semble contribuer au maintien de la pauvreté. Par ailleurs, l'État doit fournir des ressources et des services visant la réduction, voire l'éradication, de la pauvreté. Or, au NouveauBrunswick, l'aide sociale et le salaire minimum — parmi les plus bas au Canada demeurent insuffisants, laissant les femmes, entre autres, au-dessous du seuil de pauvreté (MacEwen et Saulnier, 2011). Par surcroît, des décisions gouvernementales, telles que l'abolition du Conseil consultatif du Nouveau-Brunswick sur la condition de la femme, le refus à répétition d'adopter une loi sur l'équité salariale dans le secteur privé, et les rares et faibles augmentations du salaire minimum et de l'aide au revenu, constituent des exemples concrets de la responsabilité gouvernementale dans le maintien d'une approche résiduelle en matière de pauvreté, contribuant à agrandir les trous dans le filet de sécurité sociale. Ainsi, « la pauvreté, l'écart croissant entre les riches et les pauvres, et la marginalisation continue de groupes de personnes dans nos collectivités grugent le tissu social de façon insidieuse et cela nous affecte tous" (MacEwen et Saulnier, 2011, p. 10).

Pour réellement faire sens des effets de la pauvreté dans la vie des femmes, il est nécessaire d'écouter leur voix et de valoriser leur discours pour comprendre leur trajectoire, voire rendre visibles leur vécu et leur expérience. Cette façon de faire permet de refléter la 
singularité, soit la diversité des expériences, la complexité, à savoir les intersections entre les diverses dimensions de la vie de ces femmes, et la marginalité de leur vécu, associée à leur expérience de pauvreté, à leur isolement et à l'invisibilisation de leurs conditions d'existence (Krumer-Nevo, 2005).

De manière inspirante et ingénieuse, des femmes noires états-uniennes se sont servies de la courtepointe pour se raconter, pour exister, pour pérenniser en quelque sorte leur histoire (Aptheker, 1989). Le recours à cette métaphore dans la présente recherche a servi aussi à raconter et à illustrer les pratiques quotidiennes de femmes en situation de pauvreté et de santé fragile pour mieux saisir leurs stratégies de survie et le sens de leur existence. La courtepointe comme cadre d'analyse offre l'avantage de rendre visible l'invisible, de valoriser la contribution de ces femmes à leur vie personnelle, familiale et communautaire, de les considérer comme sujets, voire actrices, de leur vie. Or, une prise de conscience de ce que la courtepointe révèle pourrait conduire à une action collective, laquelle devrait réunir les femmes et d'autres intervenantes et intervenants de la communauté qui se préoccupent de la question de la pauvreté. Pour rendre visible l'invisible, il faut d'abord créer des espaces pour que la voix de ces femmes soit entendue. Par ailleurs, l'utilisation de cette métaphore a également mis en lumière la taille importante de trous qui apparaissent dans la courtepointe. Ces trous reflètent le désengagement de l'État et une forme de désolidarisation sociale et, par conséquent, d'effritement du tissu social. Le problème qui en découle, c'est l'individualisation de la question de la pauvreté, renvoyant aux femmes la responsabilité de rapiécer les trous. En effet, au cours des vingt dernières années, l'État a transféré une large part de ses responsabilités sociales en matière de bienêtre et de sécurité sociale aux femmes individuellement (Reid et LeDrew, 2013). Or, en se mettant vraiment «les yeux devant les trous ", ce qui "saute aux yeux ", c'est que les femmes sont déjà très actives et créatives dans le rapiècement des trous laissés par l'État dans le filet de sécurité sociale, les maintenant cependant dans une situation de précarité.

\section{Notes}

1 L'inégalité du marché du travail est entre autres associée à la division sexuelle du travail, c'està-dire à un marché du travail qui est séparé et hiérarchisé, faisant en sorte que certains secteurs d'emploi sont vus comme traditionnellement féminins et moins valorisés, d'autres masculins et davantage reconnus (Kergoat, 2010).

2 La notion d'indépendance ici réfere à l'idée de rendre les assistés sociaux moins dépendants des programmes de l'État. Le gouvernement de l'époque visait à augmenter l'aptitude au travail par l'offre de programmes de retour au travail sur mesure pour les personnes perçues comme aptes au travail. 
3 Pour plus de détails historiques concernant les paiements de transfert du fédéral vers les provinces, voir le texte de Moscovitch et Thomas (2015).

\section{Bibliographie}

APTHEKER, Bettina (1989). Tapestries of Life. Women's Work, Women's Consciousness, and the Meaning of Daily Experience, Amherst, University of Massachusetts Press.

AUSTEN, Siobhan, et collab. (2015). "Care roles and employment decision-making: The effect of economic circumstance ", Journal of Industrial Relations, Vol. 57, № 5, p. 665-685.

BERTAUX, Daniel (2010). L'enquête et ses méthodes. Le récit de vie, $3^{\mathrm{e}}$ édition, Paris, Les Éditions Armand Colin.

BOISMENU, Gérard (2008). "Fédéralisme et politiques sociales au Canada ", Revue française des affaires sociales, Vol. 4, No 4, p. 7-14.

BROUSSARD, Anne C., Alfred L. JOSEPH et Marco THOMPSON (2012). «Stressors and Coping Strategies Used by Single Mothers Living in Poverty ", Affilia: Journal of Women and Social Work, Vol. 27, No 2, p. 190-204.

CAMPAGNE 2000. "La pauvreté infantile 25 ans plus tard : les mesures concrètes se font attendre depuis longtemps », réf. du 8 janvier 2016, http://www.campaign2000.ca/french/

CANTILLON, Bea (2014). "En bas de l'échelle : la difficile lutte contre la pauvreté », dans Alain Noël et Miriam Fahmy (dirs.), Miser sur l'égalité, Québec, Les Éditions Fides, p. 65-77.

CASTEL, Robert (2009). La montée des incertitudes. Travail, protections, statut de l'individu, Paris, Les Éditions du Seuil.

DEMAZIÈRE, Didier (2013). «Typologie et description. À propos de l'intelligibilité des expériences vécues ", Sociologie, Vol. 4, №3, p. 333-347.

DROLET, Marie, et collab. (2015). "Agir pour avoir accès à des services sociaux et de santé en français ", Revue canadienne de service social, Vol. 32, № 1, p. 5-26.

DROLET, Marie, et collab. (2014). «Health Services for Linguistic Minorities in a Bilingual Setting: Challenges for Bilingual Professionals ", Qualitative Health Research, Vol. 24, №3, p. 295-305.

FAHMY, Miriam, et Michel VENNE (2014). "L'égalité : une exigence démocratique », dans Alain Noël et Miriam Fahmy (dirs.), Miser sur l'égalité, Québec, Les Éditions Fides, p. 133-145.

FORGUES, Éric, et Rodrigue LANDRY (2014). L'accès aux services de santé en français et leur utilisation en contexte francophone minoritaire, Moncton, Institut canadien de recherche sur les minorités linguistiques (ICRML).

FRIEDMAN, Jennifer, et Laurel D. GRAHAM (2008). "The skills of poverty survival: One woman's life and stories ", Humanity \& Society, Vol. 32, p. 361-386. 
GOUVERNEMENT DU NOUVEAU-BRUNSWICK (2012). Profil Égalité : Femmes du NouveauBrunswick, Direction des questions féminines, Bureau du Conseil exécutif, réf. du 8 janvier 2016, http://www2.gnb.ca/content/dam/gnb/Departments/eco-bce/WI-DQF/pdf/fr/ProfilEgalite.pdf

GREEN, Autumn R. (2013). "Patchwork: Poor Women's Stories of Resewing the Shredded Safety Net ", Affilia: Journal of Women and Social Work, Vol. 28, No 1, p. 51-64.

GRELL, Paul (2015). Adolescence et suicide, Paris, Berg International Éditeurs.

HOCHSCHILD, Arlie, et Anne MACHUNG (2012). The Second Shift: Working Families and the Revolution at Home, $3^{\mathrm{e}}$ édition, New York, Penguin Books.

KAUFMANN, Jean-Claude (2006). L'enquête et ses méthodes. L'entretien compréhensif, Paris, Les Éditions Armand Colin.

KERGOAT, Danièle (2010). "Le rapport social de sexe. De la reproduction des rapports sociaux à leur subversion ", dans Annie Bidet-Mordrel (dir.), Les rapports sociaux de sexe, Paris, Presses universitaires de France, p. 44-59.

KLEIN, Juan-Luis, et Christine CHAMPAGNE (2011). " La lutte contre la pauvreté et l'exclusion sociale. Approches et stratégies institutionnelles ", dans Juan-Luis Klein et Christine Champagne (dirs.), Initiatives locales et lutte contre la pauvreté et l'exclusion, Québec, Les Presses de l'Université du Québec, p. 31-44.

KOELSCH, Lori E. (2012). "The Virtual Patchwork Quilt: A Qualitative Feminist Research Method », Qualitative Inquiry, Vol. 18, № 10, p. 823-829.

KRUMER-NEVO, Michal (2005). "Reading a Poor Woman's Life: Issues and Dilemmas », Affilia: Journal of Women and Social Work, Vol. 20, No 1, p. 87-102.

le BLANC, Guillaume (2009). L'invisibilité sociale, Paris, Les Presses Universitaires de France.

LISSE, Élisabeth (2007). « Monoparentalité et sociabilité féminine. Apprentissages du rôle de mère en cité populaire ", Ethnologie française, Vol. 37, № 4, p. 733-741.

MACEWEN, Angella, et Christine SAULNIER (2011). Coût de la pauvreté au Nouveau-Brunswick, Halifax, Centre canadien de politiques alternatives, réf. du 8 janvier 2016, https://www.policyalternatives.ca/sites/default/files/uploads/publications/Nova\%20Scotia $\% 20$ Office/2011/09/Cost\%20of\%20Povery\%20in\%20NB\%20FRENCH.pdf

MADANIPOUR, Ali, Mark SHUCKSMITH et Hilary TALBOT (2015). "Concepts of poverty and social exclusion in Europe ", Local Economy, Vol. 30, № 7, p. 1-21.

MCALL, Christopher, et collab. (2012). Au-delà du préjugé : Trajectoires de vie, pauvreté et santé, Québec, Les Presses de l'Université du Québec. 
MCALL, Christopher (2008). "Trajectoires de vie, rapports sociaux et production de la pauvreté ", dans Vivianne Châtel et Shirley Roy (dirs.), Penser la vulnérabilité. Visages de la fragilisation du social, Québec, Les Presses de l'Université du Québec, p. 93-123.

MILBOURNE, Paul (2014). «Poverty, Place, and Rurality: Material and Sociocultural Disconnections », Environment and Planning A, Vol. 46, p. 566-580.

MOREAU, André C., Stéphane SAVRIAMA et Francine A. MAJOR (2013). "Littératie en santé : conduites et gestion de soins chez les personnes ayant des compétences réduites en littératie », Globe: revue internationale d'études québécoises, Vol. 16, № 1, p. 91-105.

MOREL, Sylvie (2002). Modèle du workfare ou modèle de l'insertion? La transformation de l'assistance sociale au Canada et au Québec, Ottawa, Condition féminine Canada.

MOSCOVITCH, Allan, et Ginette THOMAS (2015). « Une nouvelle loi sur l'action sociale pour le Canada ", Travail social canadien, Vol. 17, № 1 p. 91-128.

NAYAR, Preethy, et collab. (2012). « Preventable Hospitalizations: Does Rurality or Non-Physician Clinician Supply Matter? ", J Community Health, Vol. 37, p. 487-494.

NUTBEAM, Don (2008). "The evolving concept of health literacy ", Social Science \& Medecine, Vol. 67, p. 2072-2078.

OFFER, Shira (2012). "The burden of reciprocity: Processes of exclusion and withdrawal from personal networks among low-income families ", Current Sociology, Vol. 60, № 6, p. 788-805.

OLLIVIER, Michèle, et Manon TREMBLAY (2000). Questionnements féministes et méthodologie de la recherche, Paris, Les Éditions l'Harmattan.

PAILLÉ, Pierre, et Alex MUCCHIELLI (2012). L'analyse qualitative en sciences humaines et sociales, $3^{e}$ édition, Paris, Les Éditions Armand Colin.

PAPERMAN, Patricia (2015). "L'éthique du care et les voix différentes de l'enquête ", Recherches féministes, Vol. 28, No 1, p. 29-44.

PETERSON Lars E., et David G. LITAKER (2010). «Country-level poverty is equally associated with unmet health care needs in rural and urban settings ", The Journal of Rural Health, Vol. 26, p. 373-382.

PROVENCHER, Ysabel (2004). "L'aide sociale au Nouveau-Brunswick. Évolution historique et perspectives contemporaines", Revue canadienne de politiques sociales, Vol. 54, p. 19-33.

RAÏQ, Hicham, et Charles PLANTE (2013). «Trajectoires de pauvreté et monoparentalité: le Québec dans une perspective comparative ", Sociologie et sociétés, Vol. 45, № 1, p. 67-90.

RAPHAEL, Dennis (2010). Health and Illness: About Canada, Winnipeg, Fernwood Publishing.

REID, Colleen, et Robin A. LEDREW (2013). "The Burden of Being 'Employable': Underpaid and Unpaid Work and Women's Health ", Affilia: Journal of Women and Social Work, Vol. 28, № 1, p. 79-93. 
REID, Colleen, et Allison TOM (2006). "Poor Women's Discourses of Legitimacy, Poverty, and Health ", Gender and Society, Vol. 20, №3, p. 402-421.

RÉSEAU DE DÉVELOPPEMENT ÉCONOMIQUE ET D'EMPLOYABILITÉ DU CANADA (2006). "Profils socio-économiques du Nouveau-Brunswick», réf. du 15 janvier 2016, http://www. $\mathrm{rdee.ca/statistique/fr/nouveau-brunswick/index.php}$

ROSANVALLON, Pierre (1992). La crise de l'État-providence, Paris, Les Éditions du Seuil.

SAVOIE, Lise (2011). Comprendre la trajectoire sociale de femmes francophones du Nouveau-Brunswick ayant participé à un processus d'alphabétisation : une perspective féministe, [thèse de doctorat], Université de Moncton.

SAVOIE, Lise, et Jeanne d'Arc GAUDET (2013). "Réussir son insertion professionnelle : une analyse féministe de trajectoires sociales de femmes qui se sont engagées dans un processus d'alphabétisation ", Recherches féministes, Vol. 26, № 1, p. 31-48.

SAVOIE, Lise, et collab. (2016). "Les femmes en situation de pauvreté: prendre en charge sa santé en contexte de ruralité ", Intervention, № 143, p. 15-31.

SHARMA, Raghubar D. (2012). Poverty in Canada, Don Mills, Oxford Press.

SINGH, Anneliese A. (2010). "Teaching Social Justice Advocacy Using the Metaphor of a Quilt ", Psychology of Women Quarterly, Vol. 34, No 4, p. 550-553.

STEPHENS, Nicole M., Jessica S. CAMERON et Sarah S. M. TOWNSEND (2014). «Lower Social Class Does Not (Always) Mean Greater Interdependance: Women in Poverty Have Fewer Social Resources Than Working-Class Women », Journal of Cross-Cultural Psychology, Vol. 45, № 7, p. 1061-1073.

WATTIS, Louise, et Liz JAMES (2013). « Exploring order and disorder: Women's experiences balancing work and care », European Journal of Women's Studies, Vol. 20, № 3, p. 264-278.

WILKINSON, Richard, et Kate PICKETT (2013). L'égalité, c'est mieux : pourquoi les écarts de richesse ruinent nos sociétés, Montréal, Les éditions Éco-Société.

YEANDLE, Sue, et Viktoria JOYNES (2012). «Challenges in combining work and care: Evidence from investigating women's work in Leeds ", Local Economy, Vol. 27, № 8, p. 816-830.

YEROCHEWSKI, Carole, et Francis FORTIER (2013). «Actrices des mutations ou responsables de leur précarité? Travailleuses pauvres et politiques publiques au Québec », Revue multidisciplinaire sur l'emploi, le syndicalisme et le travail, Vol. 8, № 2, p. 35-60.

YOUNG, Robert A. (2001). "Le programme Chances égales pour tous : une vue d'ensemble », dans L’ère Louis J. Robichaud, 1960-1970 : Actes du colloque, Collection Maritimes, Institut canadien de recherche sur le développement régional.

ZILANAWALA, Afshin (2014). "Women's Time Poverty and Family Structure: Differences by Parenthood and Employment ", Journal of Family Issues, p. 1-24. 